\title{
Genetic Structure of Populations of the Wheat Sharp Eyespot Pathogen Rhizoctonia cerealis Anastomosis Group D Subgroup I in China
}

\author{
Wei Li, Yingpeng Guo, Aixiang Zhang, and Huaigu Chen
}

Institute of Plant Protection, Jiangsu Academy of Agricultural Sciences, Nanjing 210014, People’s Republic of China. Accepted for publication 5 October 2016.

\begin{abstract}
Sharp eyespot on wheat is caused by Rhizoctonia cerealis anastomosis group D subgroup I (AG-DI) and is an economically important stem-base disease of wheat in temperate regions worldwide. However, the understanding about the field population structure of $R$. cerealis is limited. In this study, the genetic structure of four wheat-infecting populations in China was investigated using six microsatellite markers characterized from the transcriptome data of $R$. cerealis AG-DI. A total of 173 unique genotypes were identified among 235 fungal isolates. Departure from Hardy-Weinberg equilibrium, a significant degree of inbreeding, and a significant deficit in heterozygotes indicated a nonrandom mating pattern. Combining the low to

diversity and low to moderate clonal fractions, sexual reproduction probably existed, but the asexual reproduction should be the predominant reproductive mode. Structural analysis showed three gene pools among the four populations, which indicated the existence of three evolutionary origins of $R$. cerealis AGDI. The long-distance movement of contaminated material, especially the infected seed, might have caused the moderate gene flow among these populations, which was consistent with the high differentiation among these populations. Overall, the genetic characteristics of the populations suggested a moderate evolutionary potential for $R$. cerealis AG-DI in China.
\end{abstract} intermediate degrees of gametic disequilibrium, although with high genotypic
Additional keywords: population genetics.
Soilborne basidiomycetous fungus Rhizoctonia cerealis Van der Hoeven (teleomorph: Ceratobasidium cereale Murray and Burpee), which belongs to the binucleate Rhizoctonia anastomosis group D (AG-D), is the causal pathogen of foot rot, root rot, and damping-off diseases in several crops (González García et al. 2006; Lipps and Herr 1982). The AG-D subgroup I (AG-DI) can cause sharp eyespot on wheat and yellow patch on grasses (Hayakawa et al. 2006; Toda et al. 1999). The sharp eyespot caused by $R$. cerealis AG-DI is a stem-base wheat disease that is widely distributed in temperate wheat-growing regions worldwide (Hamada et al. 2011). In China, sharp eyespot has been a highly economically important wheat disease for many decades, especially in the lower reaches of the Yangtze River and the Yellow Huai River basins (Chen et al. 2009). In 2005, 8.1 million ha of winter wheat was affected by $R$. cerealis, demonstrating the importance and urgency of preventing and controlling this disease (McBeath and McBeath 2010). Wheat cultivars resistant to sharp eyespot can be used to prevent this disease. However, few wheat cultivars demonstrate moderate resistance, and highly resistant cultivars are rare (Chen et al. 2013). Fungicide seed coating and spraying at early jointing stage remain the main control strategies, despite the problem of increasing fungicide residues in crops and in the environment. The potential risk of the emergence of pathogen populations resistant to fungicides is also the concern, although no resistant isolates of $R$. cerealis have been detected in the field until now (Sun et al. 2015).

$R$. cerealis AG-DI does not form asexual spores, and its sexual stage is probably rare in nature (González García et al. 2006; Hayakawa et al. 2006). This fungus exists over summer or winter as a vegetative mycelium or sclerotium in plant debris or soil. Mycelium or germinating sclerotium serves as a primary inoculum

Corresponding author: H. Chen; E-mail address: huaigu@jaas.ac.cn

(C) 2017 The American Phytopathological Society and infects wheat before winter or the coming spring (Hamada et al. 2011; Lipps and Herr 1982). By using rDNA-internal transcribed spacer (ITS) sequences, preliminary studies have provided evidence for the heterogeneity of the ITS sequences and the evolution of the rDNA of $R$. cerealis AG-DI in China (Li et al. 2014).

Despite the high yield losses caused by $R$. cerealis on wheat globally, the population biology of this fungus remains poorly understood. The population genetics of multinucleate $R$. solani (teleomorph: Thanatephorus cucumeris) has recently been studied using the microsatellite markers developed from enriched genomic libraries (Zala et al. 2008) or draft genome (Ferrucho et al. 2009). Among the different strains investigated were the rice-, soybean-, maize-, and signalgrass-infecting $R$. solani AG-1 IA (Bernardes-deAssis et al. 2008, 2009; Chavarro Mesa et al. 2015; Ciampi et al. 2008; Padasht-Dehkaei et al. 2013); the potato-infecting $R$. solani AG-3 (Ferrucho et al. 2013); and the vegetable-infecting $R$. solani AG-4 (Haratian et al. 2013). For the binucleate Rhizoctonia, only the population genetic information on $R$. oryzae-sativae (teleomorph: $C$. oryzae-sativae, under AG-B), which is the causative pathogen of the rice aggregate sheath spot in California and Latin America, is available to date (Chaijuckam et al. 2010; Pereira et al. 2016).

No polymorphic microsatellite markers are currently available for population genetic studies with $R$. cerealis. In pathogenic fungi, microsatellites or tandem simple-sequence repeat (SSR) markers become powerful tools for population structure studies, particularly in understanding the evolutionary potential of populations and making predictions regarding the relative risks posed by pathogens in terms of fungicidal applications and breakdown of resistance genes (McDonald and Linde 2002). The present study aimed to (i) develop a repertoire of primer pairs that can amplify polymorphic microsatellite loci in $R$. cerealis AG-DI and employ these SSR primers to analyze the population genetics of $R$. cerealis AG-DI populations in China, (ii) measure the genetic and genotypic diversity in $R$. cerealis AG-DI populations infecting wheat, (iii) infer the predominant reproductive mode in these populations, 
and (iv) determine the occurrence and the magnitude of gene flow among populations.

\section{MATERIALS AND METHODS}

Fungal isolates. In 2010, wheat samples showing typical symptoms of sharp eyespot were collected from four different fields in China, namely, Bengbu in the Anhui Province (BB; $\left.32.55^{\circ} \mathrm{N}, 120.21^{\circ} \mathrm{E}\right)$, Linyi in the Shandong Province (LY; $\left.34.88^{\circ} \mathrm{N}, 118.17^{\circ} \mathrm{E}\right)$, Jiangyan in the Jiangsu Province (JY; $34.25^{\circ} \mathrm{N}, 116.21^{\circ} \mathrm{E}$ ), and Shangqiu in the Henan Province (SQ; $33.04^{\circ} \mathrm{N}, 117.38^{\circ} \mathrm{E}$ ). From each field, a total of 50 to 80 infected samples were collected. These samples were separated from one another by at least $10 \mathrm{~m}$ along each transect, and seven to nine transects per field were carried out. The infected stems were cut into 5- to 10-mm-long segments; processed with $75 \%$ alcohol, $\mathrm{NaOCl}(1 \%$ available $\mathrm{Cl})$, and sterile water; placed on petri plates containing potato dextrose agar (PDA) medium; and then incubated at $25^{\circ} \mathrm{C}$ in the dark. After 2 to 3 days of incubation, hyphal tips from the colonies of $R$. cerealis AG-DI were transferred to fresh PDA plates. From each field population, 51 to 70 isolates were obtained. To determine the AG of the isolates, a pair of primers developed from the $t u b B$ gene of $R$. cerealis AG-DI were used to amplify specific segments: RtubF4 (5'-CCT AAA TGA GTC TGG AGTAAG TC-3') and RtubR4 (5'-GCT AGT GCG GTC AAT GTA TAG-3') (Guo et al. 2012).

Microsatellite markers. In our previous study, we obtained the transcriptome (mRNA) of $R$. cerealis AG-DI strain R0301, which contained 21,029 unigenes with lengths of 200 to $15,995 \mathrm{bp}$ (Ren et al. 2013) (W. Li, unpublished data). The unigene sequences were screened for microsatellites by using Magellan software (Lim et al. 2004). All possible mono- to hexa-nucleotide motifs of five or more repeats in the transcriptome database were identified. Magellan was also designed to list the locations of all microsatellites found in each unigene sequence, thereby allowing easy-to-design primers to amplify selected microsatellite loci based on the surrounding sequences. The primer pairs were designed using Primer Premier Version 5.0 (Premier Biosoft International, Palo Alto, CA) and synthesized by Genscript (Genscript Biotech, Nanjing, China).

To test the polymorphism of the microsatellite markers, $15 R$. cerealis AG-DI isolates from several areas of China were randomly selected. Polymerase chain reactions (PCRs) were performed in a total of $20 \mu \mathrm{l}$ volumes with $1 \mu \mathrm{l}$ of genomic DNA (5 to $10 \mathrm{ng}$ of final concentration), $5 \mu \mathrm{l}$ of $10 \times$ reaction buffer $(500 \mathrm{mM} \mathrm{KCl}, 15 \mathrm{mM}$ $\mathrm{MgCl}_{2}, 100 \mathrm{mM}$ Tris-HCl, $\mathrm{pH} 8.8$ ), $0.3 \mu \mathrm{M}$ of each primer, $2 \mu \mathrm{l}$ of dNTP mixture (each $2.5 \mathrm{mM}$ ), and $1 \mathrm{U}$ of Taq polymerase (TaKaRa). Reactions were conducted in an S1000 TM thermal cycler (Bio-Rad) programmed for an initial incubation of $94^{\circ} \mathrm{C}$ for $5 \mathrm{~min}$, followed by 35 cycles at $94^{\circ} \mathrm{C}$ for $30 \mathrm{~s}, 58^{\circ} \mathrm{C}$ or $62^{\circ} \mathrm{C}$ for $30 \mathrm{~s}$, and $72^{\circ} \mathrm{C}$ for $30 \mathrm{~s}$. A final extension reaction was conducted at $72^{\circ} \mathrm{C}$ for $10 \mathrm{~min}$. Amplified products were analyzed by electrophoresis on $2 \%$ agarose and $8 \%$ polyacrylamide gels. Some of the PCR products amplified using high-fidelity polymerase (PrimeSTAR HS DNA Polymerase, TaKaRa) were ligated with pSIMLPE-18 EcoRV/BAP vector (TaKaRa), amplified in Escherichia coli JM109, and sequenced in Genscript (Genscript Biotech) to ensure the accuracy of the type and repeat number of the motifs.

The forward primers of the final microsatellite loci were labeled with 6-carboxyfluorescein (6-FAM) fluorescent dyes (Genscript Biotech). PCR products were separated on an ABI3730 Genetic Analyzer by using GeneScan 600 LIZ as a size standard (Applied Biosystems). The size of the fragments for each SSR was determined using GeneMapper software (Applied Biosystems), adjusted manually if necessary, and reported in Excel.

Microsatellite data analyses. $R$. cerealis AG-DI is a dikaryon (Li et al. 2014); thus, we assumed that the genetic conditions of all of the isolates used in this study were similar to a diploid organism. Data on the range of repeats of each locus, number of alleles per locus, identification of private alleles, and allelic frequencies were assessed using CONVERT version 1.31 (Glaubitz 2004).

Genotype diversity. The program GenoType (Meirmans and Van Tienderen 2004) was used to determine the multilocus microsatellite genotype (MLMG) of each strain. Isolates with the same MLMG were treated as clones. Several indices of clonal diversity were estimated for each population by using GenoDive (Meirmans and Van Tienderen 2004). These indices included (i) the number of genotypes per population; (ii) the clonal fraction (or the proportion of fungal isolates originating from asexual reproduction), calculated as 1 - [(number of different genotypes)/(total number of isolates)] (Zhan et al. 2003); and (iii) both Stoddart and Taylor's genotypic diversity and its evenness. Stoddart and Taylor's genotypic diversity is expressed as $G_{0}=1 / \Sigma p_{i}{ }^{2}$, where $p_{i}$ is the frequency of the $i$ th genotype (Stoddart and Taylor 1988), and its evenness is expressed as $G_{0}$ scaled by the maximum number of expected genotypes. The latter is an indicator of how evenly the genotypes are distributed over the population. By using a pairwise bootstrap test, we calculated whether pairs of populations differed in their clonal diversity indices. In this process, the individuals were resampled from the populations, and the diversity indices were compared after every replicate (Manly 1991). A total of 1,000 permutations with subsampling were employed to match the size of the smallest population (Grünwald et al. 2003). For all subsequent analyses, clone-corrected datasets were used.

Genetic diversity and differentiation among geographical populations. Genetic diversity was quantified on the basis of allelic richness and expected heterozygosity (HE) using FSTAT 2.9.3 (Goudet 1995). Allelic richness was estimated in accordance with the method of El Mousadik and Petit (1996) to determine the mean number of alleles per locus. HE was estimated in accordance with Nei's formula as follows: $n /(n-1) \times\left(1-\Sigma i p i^{2}\right)$, where $p$ is the observed frequency of the $i$ th allele and $n$ is the sample size (Nei 1978). A bootstrapping approach based on 1,000 permutations was also performed using FSTAT 2.9.3 (Goudet 1995) to test whether the populations differed in genetic diversity and allelic richness.

The degree of population subdivision and the distribution of genetic diversity among populations of $R$. cerealis AG-DI were estimated by pairwise comparisons of populations through analysis of molecular variance (AMOVA) with the program Arlequin 3.5 (Excoffier et al. 2005). A hierarchical analysis of variance was used to partition the covariance components into those among populations and those within populations. The pairwise fixation index $\left(R_{\mathrm{ST}}\right)$ between groups of populations was calculated to examine population differentiation. The significance of both analyses was determined using 1,000 permutations by a nonparametric approach in Arlequin 3.5 (Excoffier et al. 2005).

Reproductive mode: Hardy-Weinberg equilibrium (HWE) and gametic equilibrium (GE) tests. HWE analysis was performed using Fisher's exact test with a Markov chain (chain length: 1,000,000; dememorization: 100,000) by employing Arlequin 3.5 (Excoffier et al. 2005). GE was assessed using a multilocus association test (Brown et al. 1980). A hypothesis proposing that genotypes at one locus are independent from genotypes at another locus was evaluated using the Markov chain Monte Carlo (MCMC) algorithm (with 1,000 batches and 1,000 iterations per batch), which was implemented in GenePop 4.0.11 (http://genepop.curtin.edu.au/) (Raymond and Rousset 1995). Using Multilocus v1.3 (Agapow and Burt 2001), we measured the multilocus index of association $\left(I_{A}\right)$ (Smith et al. 1993). The inbreeding coefficient $R_{\mathrm{IS}}$ was calculated using Arlequin 3.5 (Excoffier et al. 2005) with 1,000 permutations of the dataset.

Test for admixture or hidden population structure. The admixture of the population is a possible cause of observed departures from HWE and GE. A clustering method based on the Bayesian model was implemented in Structure 2.3.4 to test the pattern of population structure and detection of probable introgression (Pritchard et al. 2000). In this process, an admixture model with correlated allele frequencies was adopted. To achieve the optimum $K$ value, five replicates were run, 10,000 burn-in 
periods were employed, and 100,000 MCMC replications for data collection were conducted (Falush et al. 2003). The optimum number of clusters $(\Delta K)$ was calculated between $K=1$ and $K=7$, in accordance with the simulation method of Evanno et al. (2005) using Structure Harvester v0.6.8 (https://users.soe.ucsc.edu/ dearl/ software/struct_harvest/) (Earl 2012). To facilitate interpretation, CLUMPP (http://web.stanford.edu/group/rosenberglab/clumpp.html) (Jakobsson and Rosenberg 2007) was used to align multiple replicate analyses of the dataset from Structure Harvester.

Historical migration. The effective population sizes $(\theta)$ and historical migration rates $(4 \mathrm{Nm})$ among the four populations were estimated using a maximum likelihood test with MIGRATE 3.2 (Beerli and Felsenstein 2001). MIGRATE is based on a coalescent theory to estimate $\theta$ values and the migration matrix (Beerli and Felsenstein 2001). This program can be used to infer the asymmetrical gene flow among populations by analyzing the microsatellite data with Brownian motion and assuming a stepwise mutation model. A single run consisted of five replicates with 10 initial short chains and 3 long final chains, as well as a static heating scheme with four temperatures $(1.0,1.3,2.6$, and 3.9). For the short chain, the recorded steps were 1,000 , the increment was 20 , and the visited genealogies were 20,000 . For the long chain, the recorded steps were 5,000, the increment was 20 , and the visited genealogies were 100,000. The number of discarded trees per short and long chains was 10,000. The confidence interval for $\theta$ and migration parameter $M$ was calculated using a percentile approach.

\section{RESULTS}

Microsatellite markers and fungal populations. Among 21,029 unigene sequences, we found 220 di-, 112 tri-, and 1 hexanucleotide microsatellite motifs (Table 1). No tetra- and pentanucleotide motifs were found in these sequences (Table 1). However, most of the microsatellite loci were located in the $5^{\prime}$ or $3^{\prime}$ end of the unigene sequences, on which no primers can be designed. Given these sequences, 24 microsatellite loci, comprising seven di-, nine tri-, one hexa-, and seven compound-nucleotide

TABLE 1. Number of microsatellite motifs in the transcriptome of Rhizoctonia cerealis anastomosis group D subgroup I

\begin{tabular}{lrrrrrrr}
\hline \multicolumn{7}{c}{ Number of repeats } & \\
\cline { 2 - 6 } Motif & \multicolumn{1}{c}{5} & \multicolumn{1}{c}{6} & \multicolumn{1}{c}{7} & 8 & 9 & $>10$ & Sum of motifs \\
\hline Mono & 35,472 & 7,937 & 1,485 & 276 & 89 & 114 & 45,373 \\
Di & 189 & 27 & 3 & 1 & 0 & 0 & 220 \\
Tri & 85 & 17 & 5 & 3 & 0 & 2 & 112 \\
Tetra & 0 & 0 & 0 & 0 & 0 & 0 & 0 \\
Penta & 0 & 0 & 0 & 0 & 0 & 0 & 0 \\
Hexa & 0 & 0 & 0 & 1 & 0 & 0 & 1 \\
\hline
\end{tabular}

microsatellite motifs, were identified and selected to develop primer pairs. Among these microsatellite loci, 17 were successfully amplified from the $R$. cerealis AG-DI strains, and 11 exhibited a size polymorphism within different strains. To ensure the accuracy of the type and repeat number of the motifs, these 11 microsatellite loci were amplified using a high-fidelity polymerase, and the PCR products were sequenced and analyzed. Finally, six microsatellite loci were confirmed, of which two were dinucleotide motifs with 2 to 12 repeat numbers, three were trinucleotide motifs with 3 to 12 repeat numbers, and 1 was a hexa-nucleotide motif with one to eight repeat numbers. The primer sequences and the allele size range are listed in Table 2.

A total of 235 isolates from four geographical populations were analyzed in this study, of which 57 isolates were from the BB populations, 70 from the LY, 57 from the JY, and 51 from the SQ (Table 3). All isolates were identified to be $R$. cerealis AG-DI on the basis of morphological characteristics (Chen et al. 2009) and specific primer amplification RtubF/R4 (Guo et al. 2012). All the loci were polymorphic in the four populations of $R$. cerealis AG-DI with high genetic diversity (Table 2). Two to four private alleles were found in the BB, LY, and JY populations, whereas no private alleles were found in the SQ population (Table 3).

Microsatellite information content and genetic and geno typic diversity. A total of 173 different genotypes were detected among the four populations of $R$. cerealis AG-DI. Overall, only two genotypes were shared between the BB and LY populations. Small to intermediate clonal fractions were noted for all populations. The smallest clonal fraction was 0.12 in the SQ population, which exhibited the highest genotypic diversity (37.7) and evenly distributed genotypes (evenness $=0.84)$ (Table 3$)$. Intermediate clonal fractions ( 0.21 to 0.41$)$ were observed for the other three populations. LY, which is the most northern population, displayed the highest clonal fractions (0.41) and site-specific genotypes (39) and exhibited the lowest genotypic diversity (19.6) and evenness (0.48) (Table 3). The majority of the genotypes (171 of the 173) were heterozygous for at least one locus. The HE was 0.51 to 0.59 , and Nei's genetic diversity values were not significantly different $(P \leq 0.05)$ among the four populations (Table 3 ).

Population differentiation. On the basis of the AMOVA esti mates, significant differentiation existed among the populations. The variations among populations only represented $3.69 \%$ of the total variance. By contrast, the variation within individuals accounted for $69.13 \%$ of the total variance (Table 4). Pairwise analysis of population differentiation also indicated that these four populations were significantly differentiated, except the differentiation between the $\mathrm{BB}$ and $\mathrm{LY}$ populations $\left(R_{\mathrm{ST}}=0.008, P=0.12\right.$, insignificant). The highest $R_{\mathrm{ST}}$ value was found between the $\mathrm{BB}$ and JY populations $\left(R_{\mathrm{ST}}=0.064, P=0.0\right)$, and the other $R_{\mathrm{ST}}$ values were 0.038 to $0.045(P=0.0)$ (Table 5).

TABLE 2. Description of primers, conditions of annealing temperature for PCR amplification, allele size, and number of repeats

\begin{tabular}{|c|c|c|c|c|c|c|c|c|c|c|}
\hline \multirow{2}{*}{$\begin{array}{l}\text { Marker } \\
\text { name }\end{array}$} & \multirow[b]{2}{*}{ Repeat motif } & & \multirow[b]{2}{*}{ Primers $\left(5^{\prime}-3^{\prime}\right)$} & \multirow[b]{2}{*}{$\operatorname{Tm}\left({ }^{\circ} \mathrm{C}\right)$} & \multirow{2}{*}{$\begin{array}{l}\text { Allele size } \\
\text { range (bp) }\end{array}$} & \multirow{2}{*}{$\begin{array}{l}\text { Number } \\
\text { of alleles }\end{array}$} & \multicolumn{4}{|c|}{ Gene diversity ${ }^{z}$} \\
\hline & & & & & & & $\mathrm{BB}$ & LY & JY & SQ \\
\hline \multirow[t]{2}{*}{$\mathrm{R} 4$} & $(\mathrm{AGA}) 5-9$ & $\mathrm{R} 4-\mathrm{u}$ & AGTAGAGGTGATGGAGGAAG & 58 & $275-287$ & 5 & 0.69 & 0.73 & 0.69 & 0.71 \\
\hline & & R4-d & CTGATGACGCTGTATGGTAT & & & & & & & \\
\hline R5 & & R5-d & GTCCCAACAACGCTCCTCAT & & & & & & & \\
\hline \multirow[t]{2}{*}{ R14 } & (GTG)4-12 & $\mathrm{R} 14-\mathrm{u}$ & CATCCCGTTGTGTGCCATTG & 62 & $315-339$ & 9 & 0.82 & 0.76 & 0.67 & 0.85 \\
\hline & & R14-d & GTCTCTCTCACCACCGCAGT & & & & & & & \\
\hline $\mathrm{R} 21$ & & R21-d & CTCCAGGCGTTACGAATA & & & & & & & \\
\hline \multirow[t]{2}{*}{ R24 } & (TG)4-12 & $\mathrm{R} 24-\mathrm{u}$ & GCTACTAGGGTCCTTTCA & 58 & $308-324$ & 9 & 0.44 & 0.52 & 0.72 & 0.73 \\
\hline & & R24-d & AAACCAACGGAGTTACGG & & & & & & & \\
\hline
\end{tabular}

${ }^{\mathrm{z}}$ Unbiased gene diversity was computed according to Nei (1978) using FSTAT version 2.9.3. 
HWE and GE tests. The BB population exhibited half of the loci in the HWE, whereas the other populations contained more than half loci out of HWE (Table 6). All four populations of $R$. cerealis AG-DI showed significant departures from HWE. Positive values of inbreeding coefficients $R_{\mathrm{IS}}$ from 0.11 to 0.31 ( $\left.P \leq 0.01\right)$ were obtained for the four populations, and this finding indicated the significant inbreeding in these populations. In the GE test, the BB $\left(I_{A}=0.071, P=0.208\right)$ and $\mathrm{LY}\left(I_{A}=0.093, P=0.147\right)$ populations were in GE. Significant $I_{A}$ was observed in the JY $\left(I_{A}=0.147, P=\right.$ $0.025)$ and SQ $\left(I_{A}=0.151, P=0.032\right)$ populations. However, 20 to $40 \%$ of locus pairs were in disequilibrium.

Test for admixture or hidden population structure. By using Structure Harvester and on the basis of $\Delta K$ value, we analyzed the optimum number of clusters. The results from Structure Harvester indicated that the highest peak of $\Delta K$ appeared when $K=3$ (Evanno et al. 2005). Given this result, we considered $K=3$ to be the supported number of populations. Subsequently, three were selected as the optimal number of groups. The subpopulation structure in 173 lines was determined as three different bar plots (Fig. 1). The diagram showing the individual assignments to the clusters revealed a clonal structure to the data. In the three-cluster model $(K=3)$, varying shades of gray represent three gene pools. All of the populations exhibited a similar cluster, with mixed individuals.

Demographic parameters and historical migration. The effective population size estimates of the BB, JY, and SQ populations were highly similar $(\theta=0.93,0.91$, and 0.94$)$, whereas that of the LY population was larger $(\theta=1.16)$ (Fig. 2). The historical migration rates provide evidence of directional gene flow among the different populations. In this study, evidence for gene flow was found among all $R$. cerealis AG-DI populations in China. Increased migration was found between the two northern populations $\left(4 N m_{\mathrm{LY} \rightarrow \mathrm{SQ}}=1.59,4 N m_{\mathrm{SQ} \rightarrow \mathrm{LY}}=2.72\right)$. Most of the exchanges of immigrants between these populations were asymmetrical. The highest asymmetric migration estimates were observed between LY and $\mathrm{BB}\left(4 N m_{\mathrm{LY} \rightarrow \mathrm{BB}}=3.33,4 N m_{\mathrm{BB} \rightarrow \mathrm{LY}}=1.11\right)$. The population that contributed the highest number of migrants to majority of the populations (except $\mathrm{JY})$ was $\mathrm{LY}\left(4 N m_{\mathrm{LY} \rightarrow \mathrm{BB}}=3.33,4 N m_{\mathrm{LY} \rightarrow \mathrm{SQ}}=\right.$
1.59) (Fig. 2). Generally, all the populations contributed migrants to other populations, and the northern populations (LY and SQ) contributed more than the southern populations did (BB and JY).

\section{DISCUSSION}

In this study, we analyzed all the microsatellite loci in the transcriptome of the $R$. cerealis AG-DI strain R0301. We also described a set of primer pairs that can amplify six polymorphic microsatellite loci in $R$. cerealis AG-DI. We used these markers to analyze four populations comprising a total of 235 isolates from China. The results indicated that the microsatellite markers developed in this study were species-specific and can be used to distinguish a large number of $R$. cerealis AG-DI genotypes. To our knowledge, this paper reports the first codominant microsatellite markers of $R$. cerealis. Therefore, this set of microsatellite markers may represent an important tool for population genetic investigations on this fungus.

$R$. cerealis AG-DI is mainly considered as an asexual fungus on wheat. Sclerotia are produced at the end of the season and infect the next wheat crop, but the sexual stage of this fungus remains unknown (González García et al. 2006; Hayakawa et al. 2006; Lipps and Herr 1982; Toda et al. 1999). Mixed reproductive modes were

TABLE 5. Fixation indices $\left(R_{\mathrm{ST}}\right)$ for pairwise comparisons between Rhizoctonia cerealis anastomosis group D subgroup I populations ${ }^{\mathrm{Z}}$

\begin{tabular}{lcccc}
\hline & \multicolumn{4}{c}{ Sum of squared size differences $\left(R_{\mathrm{ST}}\right)$} \\
\cline { 2 - 5 } Population & $\mathrm{BB}$ & $\mathrm{LY}$ & $\mathrm{JY}$ & $\mathrm{SQ}$ \\
\hline BB & - & & & \\
LY & $0.008^{\mathrm{NS}}$ & - & - & \\
JY & $0.064^{*}$ & $0.042^{*}$ & $0.045^{*}$ & - \\
SQ & $0.038^{*}$ & $0.045^{*}$ & 0.0 \\
\hline
\end{tabular}

$\mathrm{z}$ Pairwise fixation indices were computed between pairs of populations with 1,000 using Arlequin 3.5 (Excoffier et al. 2005). NS and * indicate insignificant or significant at $\alpha=0.05$.

TABLE 3. Measures of genotypic diversity in Rhizoctonia cerealis anastomosis group D subgroup I populations

\begin{tabular}{|c|c|c|c|c|c|c|c|c|c|}
\hline Population & $\begin{array}{l}\text { Sample } \\
\text { size }\end{array}$ & $\begin{array}{c}\text { Number } \\
\text { of genotypes }\end{array}$ & $\begin{array}{l}\text { Site-specific } \\
\text { genotypes }^{\mathrm{s}}\end{array}$ & $\begin{array}{l}\text { Clonal } \\
\text { fraction }\end{array}$ & $\begin{array}{l}\text { Effective number } \\
\text { of genotypes }{ }^{\mathrm{t}, \mathrm{u}}\end{array}$ & Evenness $^{\mathrm{u}, \mathrm{v}}$ & $\mathrm{HE}^{\mathrm{w}, \mathrm{x}}$ & Allelic richness ${ }^{\mathrm{x}, \mathrm{y}}$ & $\begin{array}{c}\text { Number of } \\
\text { private alleles }\end{array}$ \\
\hline $\mathrm{BB}$ & 57 & 42 & $40(2)$ & 0.26 & $31.54 \mathrm{a}$ & $0.75 \mathrm{a}$ & $0.55 \mathrm{a}$ & $5.33 \mathrm{a}$ & 2 \\
\hline LY & 70 & 41 & $39(2)$ & 0.41 & $19.60 \mathrm{~b}$ & $0.48 \mathrm{~b}$ & $0.59 \mathrm{a}$ & $6.17 \mathrm{a}$ & 3 \\
\hline JY & 57 & 45 & $45(0)$ & 0.21 & $35.70 \mathrm{ac}$ & $0.79 \mathrm{a}$ & $0.53 \mathrm{~b}$ & $5.26 \mathrm{a}$ & 4 \\
\hline SQ & 51 & 45 & $45(0)$ & 0.12 & $37.70 \mathrm{c}$ & $0.84 \mathrm{a}$ & $0.51 \mathrm{~b}$ & $4.83 \mathrm{a}$ & 0 \\
\hline Total & 235 & 173 & Overall $=$ & 0.25 & 31.1 & 0.72 & - & 5.40 & - \\
\hline
\end{tabular}

s Numbers of genotypes shared with other populations are shown in parentheses.

t Stoddart and Taylor's genotypic diversity $\left(G_{0}\right)$ (Stoddart and Taylor 1988).

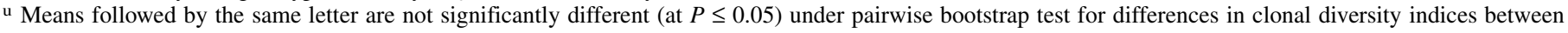
populations calculated by GenoDive (Meirmans and Van Tienderen 2004), using 1,000 permutations with subsampling to match the size of the smallest population.

v $G_{0}$ scaled by the maximum number of expected genotypes; evenness value $=1.0$ indicates that all genotypes possess equal frequencies

" Nei's unbiased gene diversity, also known as expected heterozygosity, averaged over all loci, corrected for the sample size (Nei 1978).

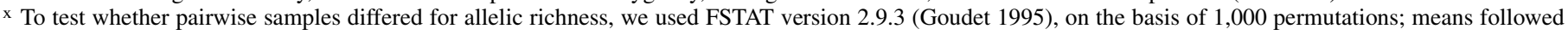
by the same letter are not significantly different $(P \leq 0.05)$.

y Calculated according to El Mousadik and Petit (1996).

$\mathrm{z}$ Alleles occurring in only one population, calculated with Convert 1.31 (Glaubitz 2004).

TABLE 4. Hierarchical distribution of gene diversity among Rhizoctonia cerealis anastomosis group D subgroup I populations ${ }^{\mathrm{z}}$

\begin{tabular}{|c|c|c|c|c|c|}
\hline \multirow[b]{2}{*}{ Source of variation } & \multicolumn{5}{|c|}{ Distance method: sum of squared size differences $\left(R_{\mathrm{ST}}\right)$} \\
\hline & Sum of squares & Variance components & Percent variation & Fixation indices & $P$ \\
\hline Among populations & 22.38 & 0.06 & 3.69 & $R_{\mathrm{IS}}=0.28$ & $0.01 *$ \\
\hline Among individuals within populations & 351.15 & 0.46 & 27.18 & $R_{\mathrm{ST}}=0.04$ & $0.02 *$ \\
\hline Within individuals & 201.50 & 1.17 & 69.13 & $R_{\mathrm{IT}}=0.31$ & 0.06 \\
\hline Total & 575.03 & 1.69 & & & \\
\hline
\end{tabular}

${ }^{\mathrm{z}}$ Analysis of molecular variance was performed using Arlequin 3.5 (Excoffier et al. 2005). * indicates significant at $\alpha=0.05$. 
reported for $R$. solani AG-1 IA, AG-3, AG-4, and $R$. oryzae-sativae (Bernardes-de-Assis et al. 2008, 2009; Chaijuckam et al. 2010; Ciampi et al. 2008; Ferrucho et al. 2013; Haratian et al. 2013; Padasht-Dehkaei et al. 2013). An increasing number of studies have documented that basidiospores may play an important role in the disease cycle and thus cause important ecological and population consequences for the Rhizoctonia fungi (Bernardes-de-Assis et al. 2008, 2009; Chaijuckam et al. 2010; Ciampi et al. 2008; Cubeta and Vilgalys 1997; Ferrucho et al. 2013; Haratian et al. 2013; Padasht-Dehkaei et al. 2013). In this study, the clonal fractions of $R$. cerealis AG-DI at 0.12 to 0.41 were unexpected results (Table 3), but most of the loci departed from HWE, with a significant degree of inbreeding, and half of the populations were in GD (Table 6). Hence, asexual reproduction may be the predominant reproduction model in $R$. cerealis AG-DI.

Sexual crossing generally causes genomic recombination in fungi (McDonald and Linde 2002; Milgroom 1996). In the asexual

TABLE 6. Hardy-Weinberg equilibrium (HWE) and gametic disequilibrium (GD) tests for Rhizoctonia cerealis anastomosis group D subgroup I populations

\begin{tabular}{|c|c|c|c|c|c|c|c|}
\hline \multirow[b]{2}{*}{ Population } & \multirow[b]{2}{*}{ Number of genotypes } & \multirow[b]{2}{*}{ Number of loci under HWEw } & \multirow[b]{2}{*}{$R_{\mathrm{IS}}{ }^{\mathrm{x}}$} & \multicolumn{4}{|c|}{ Gametic equilibrium estimates } \\
\hline & & & & $I_{A}{ }^{y}$ & $P$ value ${ }^{y}$ & Locus pairs at significant disequilibrium ${ }^{z}$ & $\%$ \\
\hline$\overline{\mathrm{BB}}$ & 42 & $3 / 6$ & 0.113 & 0.071 & 0.208 & $4 / 15$ & 26.7 \\
\hline LY & 41 & $1 / 6$ & 0.307 & 0.093 & 0.147 & $5 / 15$ & 33.3 \\
\hline SQ & 45 & $2 / 6$ & 0.203 & 0.151 & $0.032 *$ & $3 / 15$ & 20 \\
\hline
\end{tabular}

${ }^{\text {w } H W E}$ test was performed by Fisher's exact test using a Markov chain (chain length: 1,000,000; dememorization: 100,000) in Arlequin 3.5.

x Population-specific inbreeding coefficients $\left(R_{\mathrm{IS}}\right)$ and $P$ values were calculated with 1,000 permutations using Arlequin 3.5 .

${ }^{y}$ Index of association $\left(I_{A}\right)$ and $P$ values were computed in Multilocus v1.3 (Agapow and Burt 2001); the significance of $I_{A}$ was tested by comparing the observed and expected values under the null hypothesis of random mating with 1,000 randomizations. * indicates significant at $\alpha=0.05$.

z Pairs of loci at significant disequilibrium in accordance with the Fisher exact test (probability test) using a Markov chain with 1,000 batches and 1,000 iterations/ batch, implemented by GenePop 4.0, after Bonferroni correction for multiple comparisons.

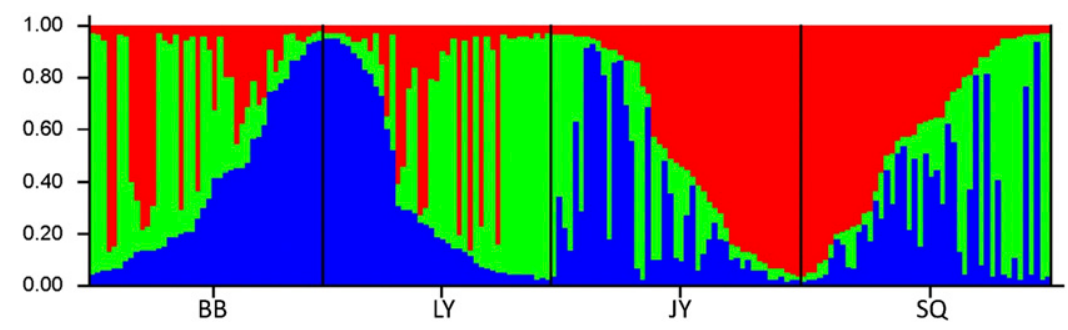

Fig. 1. Structure-inferred membership coefficient for multilocus microsatellite genotypes of the populations of Rhizoctonia cerealis anastomosis group D subgroup I in China. Each individual is represented by a thin vertical bar, which is partitioned into $K$ shaded segments that represent the estimated membership fractions of an individual in $K=3$ clusters. Black lines separate individuals of different populations. The bar length indicates its membership coefficient to the distinctly shaded populations.

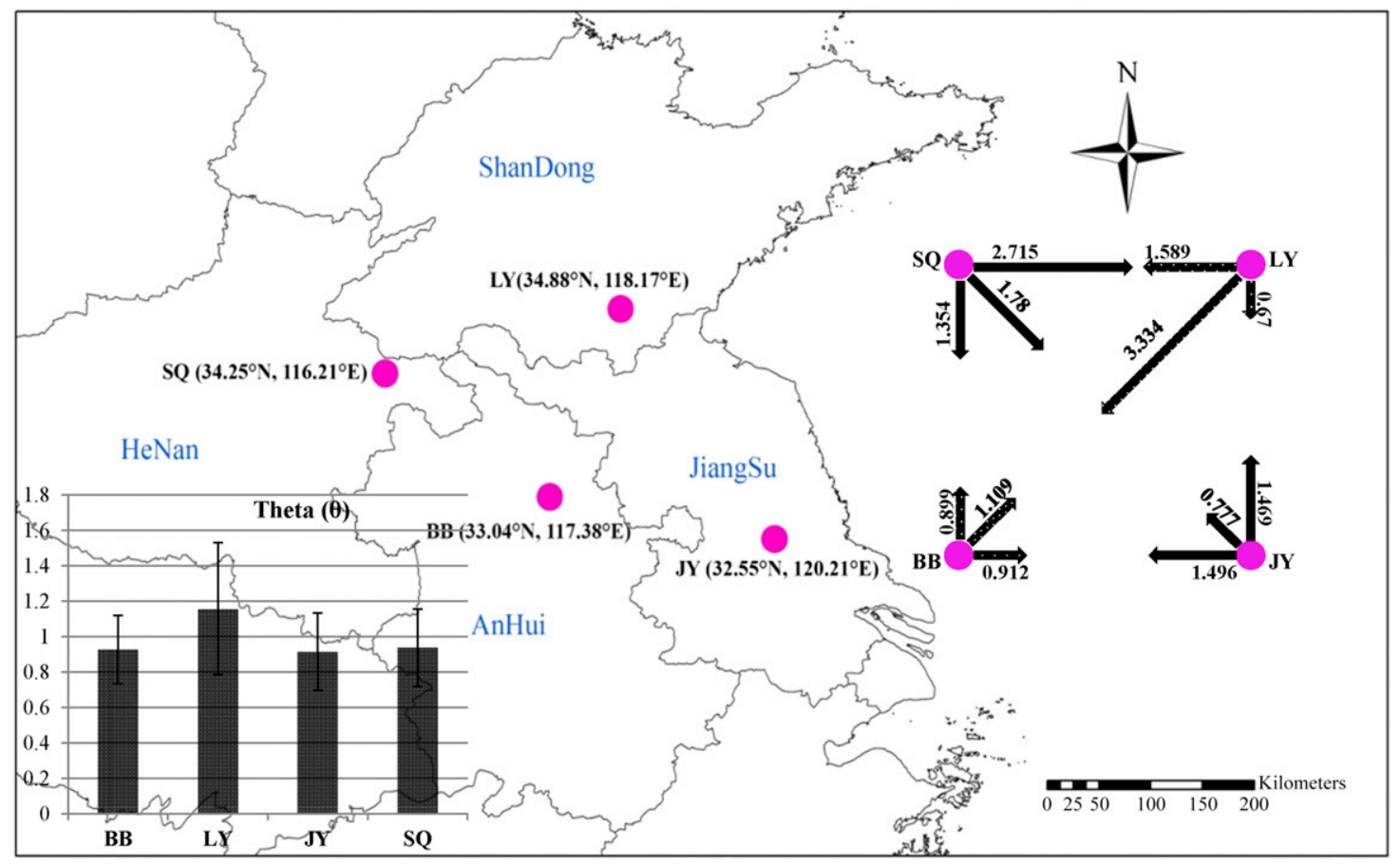

Fig. 2. Estimates of demographic parameters from the divergence among wheat-infecting populations of Rhizoctonia cerealis anastomosis group D subgroup I in China. The directions for migration between the sink and source populations are indicated by the arrows, and the migration rate per generation (4Nm) is displayed on the arrows. Estimates of the theta include $95 \%$ confidence intervals indicated as vertical bars. 
reproduction model of $R$. cerealis AG-DI, whether recombination will occur is currently unknown. By contrast, hyphal anastomosis is a common phenomenon among isolates of the same AG in the genus Rhizoctonia. Previous studies have shown the existence of two phenotypes (C2 and $\mathrm{C} 3$ ) of hyphal anastomosing reactions among different isolates belonging to the same AG (Cubeta and Vilgalys 1997). C2 involves a killing reaction and evident wall fusion, with cell death of anastomosing and adjacent cells. In this case, the somatic incompatibility response is often macroscopically visible. By contrast, C3 includes perfect fusion, with evident wall and membrane merging. The point of anastomosis is not clearly visible, and cell death is absent as these two phenotypes of interactions (C2 and C3) represent different genetic relationships among isolates (Cubeta and Vilgalys 1997; González García et al. 2006). If genomic recombination can occur through hyphal fusion in this fungus, the phenomenon should be nonrandom mating, consistent with the significant degree of inbreeding in $R$. cerealis AG-DI (Table 6). To our knowledge, no previous study has demonstrated the transmission of cytoplasmic element and nucleus via hyphal anastomosis in Rhizoctonia fungi (Cubeta and Vilgalys 1997). However, this probability cannot be ruled out, and the transfer of genetic material via hyphal anastomosis remains a possibility. This hypothesis must be further investigated and confirmed in the laboratory.

Population genetic analysis provided the resolution to infer the life histories and the evolutionary process of pathogens that shape pathogen populations in agroecosystems (McDonald and Linde 2002). Our previous study obtained 36 unique ITS sequences from $15 R$. cerealis AG-DI isolates ( $\mathrm{Li}$ et al. 2014). According to the phylogeny analysis and median-joining network, all these sequences can be divided into three groups, which implied the possibility of three evolutionary origins of this fungus (Li et al. 2014). In the current study, structure analysis showed that all of the 173 unique genotypes can be separated into three groups (Fig. 1). Although the isolates are different in the two studies, the present results suggested the existence of three gene pools in $R$. cerealis. The fact that four populations from different locations were divided to three groups, might indicate that geographic isolation did not cause reproductive isolation. And another possibility is that three evolutionary origins of this fungus existed before the geographic isolation of $R$. cerealis.

The genotypic flow in $R$. solani AG-1 IA, the causal agent of sheath blight disease in rice, is potentially mediated through the dispersal of sclerotia in irrigation water. In particular, high historical migration rates are found among rice-infecting populations from the Yangtze tributary region in China (Bernardes-de-Assis et al. 2009). For $R$. cerealis AG-DI, the sclerotia are the most important organs in summer and winter, serving as the primary inocula. This component is found between the leaf sheath and stem or within the stem lumen of the wheat. The sclerotia of $R$. cerealis AG-DI typically cannot travel long distances via irrigation water, especially in northern China. However, high historical migration rates (maximum $4 \mathrm{Nm}=3.33$ ) are also noted in these populations. In the current study, increased genetic exchange existed between the two northern populations (LY and SQ), and they contributed more migrants than the southern populations (BB and JY) did (Fig. 2). We hypothesized that the genetic flow in this area can be mainly ascribed to the human-mediated long-distance movement of the contaminated materials, such as infested seed or straw, from north to south. Considering that winter wheat is the major cultivar in northern China, similar cultivars can also be planted sometimes in the Yangtze River Basin. Thus, the pathogen can spread with the infested seed to southern areas. However, most of the southern wheat cultivars are spring wheat, and they cannot be planted in northern areas. The spread of the contaminated material through the harvester is another possible reason, but the harvest of wheat in China occurs from south to north. Compared with the spread by infested seed, this hypothesis was not significant. Thus, the seed treatment with fungicides would be useful to prevent long-distance dispersal in these areas.
This paper presents the first in-depth population genetic analysis of the wheat sharp eyespot pathogen $R$. cerealis AG-DI from China. In these populations, we found high genetic and genotypic diversity, moderate gene flow, and asexual reproduction as the predominant reproductive model with significant inbreeding. Medium to high differentiation among populations was found based on pairwise analyses of population differentiation. According to the risk model framework proposed by McDonald and Linde (2002), the population genetic characteristics of $R$. cerealis AG-DI in China indicated that this pathogen demonstrated a moderate evolutionary potential. Hence, disease management measures such as traditional fungicides and the moderate resistant wheat cultivars could still be used. At the same time, the new fungicide development and breeding new resistant varieties also need attention.

\section{ACKNOWLEDGMENTS}

This work was supported by the National Science Foundation of China (Grant 30900928), the fund earmarked for the China Agricultural Research System (CARS-3-1-17), and the National Non-Profit Industry-Funded Research and Special Projects (201503112-9).

\section{LITERATURE CITED}

Agapow, P.-M., and Burt, A. 2001. Indices of multilocus linkage disequilibrium. Mol. Ecol. Notes 1:101-102.

Beerli, P., and Felsenstein, J. 2001. Maximum likelihood estimation of a migration matrix and effective population sizes in $n$ subpopulations by using a coalescent approach. Proc. Natl. Acad. Sci. USA 98:4563-4568.

Bernardes-de-Assis, J., Peyer, P., Zala, M., Rush, M., McDonald, B. A., and Ceresini, P. C. 2008. Divergence between sympatric rice- and soybean-infecting populations of Rhizoctonia solani AG-1 IA. Phytopathology 98:1326-1333.

Bernardes-de-Assis, J., Storari, M., Zala, J., Wang, W., Jiang, W., ShiDong, L., Jin, M., McDonald, B. A., and Ceresini, P. C. 2009. Genetic structure of populations of the rice-infecting pathogen Rhizoctonia solani AG-1 IA from China. Phytopathology 99:1090-1099.

Brown, A. H. D., Feldman, M. W., and Nevo, E. 1980. Multilocus structure of natural populations of Hordeum spontaneum. Genetics 96:523-536.

Chaijuckam, P., Baek, J. M., Greer, C. A., Webster, R. K., and Davis, R. M. 2010. Population structure of Rhizoctonia oryzae-sativae in California rice fields. Phytopathology 100:502-510.

Chavarro Mesa, E., Ceresini, P. C., Ramos Molina, L. M., Pereira, D. A., Schurt, D. A., Vieira Jr, J. R., Poloni, N. M., and McDonald, B. A. 2015. The Urochloa foliar blight and collar rot pathogen Rhizoctonia solani AG-1 IA emerged in South America via a host shift from rice. Phytopathology 105:1475-1486.

Chen, J., Li, G. H., Du, Z. Y., Quan, W., Zhang, H. Y., Che, M. Z., Wang, Z., and Zhang, Z. J. 2013. Mapping of QTL conferring resistance to sharp eyespot (Rhizoctonia cerealis) in bread wheat at the adult plant growth stage. Theor. Appl. Genet. 126:2865-2878.

Chen, Y., Li, W., Zhang, X. X., Zhang, B. Q., Yu, H. S., and Chen, H. G. 2009. Composition and virulence of pathogen of wheat sharp eyespot in north latitude 33 of China. (in Chinese) J. Triticeae. Crops. 29:1110-1114.

Ciampi, M. B., Meyer, M. C., Costa, M. J. N., Zala, M., McDonald, B. A., and Ceresini, P. C. 2008. Genetic structure of populations of Rhizoctonia solani anastomosis group-1 IA from soybean in Brazil. Phytopathology 98:932-941.

Cubeta, M. A., and Vilgalys, R. 1997. Population biology of the Rhizoctonia solani complex. Phytopathology 87:480-484.

Earl, D. A. 2012. STRUCTURE HARVESTER: A website and program for visualizing STRUCTURE output and implementing the Evanno method. Conserv. Genet. Resour. 4:359-361.

El Mousadik, A., and Petit, R. J. 1996. High level of genetic differentiation for allelic richness among populations of the argan tree (Argania spinosa (L.) Skeels) endemic to Morocco. Theor. Appl. Genet. 92:832-839.

Evanno, G., Regnaut, S., and Goudet, J. 2005. Detecting the number of clusters of individuals using the software STRUCTURE: A simulation study. Mol. Ecol. 14:2611-2620

Excoffier, L., Laval, G., and Schneider, S. 2005. Arlequin (version 3.0): An integrated software package for population genetics data analysis. Evol. Bioinform. Online 1:47-50.

Falush, D., Stephens, M., and Pritchard, J. K. 2003. Inference of population structure using multilocus genotype data: Linked loci and correlated allele frequencies. Genetics 164:1567-1587.

Ferrucho, R. L., Ceresini, P. C., Ramirez-Escobar, U. M., McDonald, B. A., Cubeta, M. A., and García-Domínguez, C. 2013. The population genetic 
structure of Rhizoctonia solani AG-3PT from potato in the Colombian Andes. Phytopathology 103:862-869.

Ferrucho, R. L., Zala, M., Zhang, Z., Cubeta, M. A., Garcia-Dominguez, C., and Ceresini, P. C. 2009. Highly polymorphic in silico-derived microsatellite loci in the potato-infecting fungal pathogen Rhizoctonia solani anastomosis group 3 from the Colombian Andes. Mol. Ecol. Resour. 9:1013-1016.

Glaubitz, J. C. 2004. Convert: A user-friendly program to reformat diploid genotypic data for commonly used population genetic software packages. Mol. Ecol. Notes 4:309-310.

González García, V., Portal Onco, M. A., and Rubio Susan, V. 2006. Review: Biology and systematics of the form genus Rhizoctonia. Span. J. Agric. Res. 4:55-79.

Goudet, J. 1995. FSTAT (version 1.2): A computer program to calculate F-statistics. J. Hered. 86:485-486.

Grünwald, N. J., Goodwin, S. B., Milgroom, M. G., and Fry, W. E. 2003. Analysis of genotypic diversity data for populations of microorganisms. Phytopathology 93:738-746.

Guo, Y. P., Li, W., Sun, H. Y., Wang, N., Yu, H. S., and Chen, H. G. 2012. Detection and quantification of Rhizoctonia cerealis in soil using real-time PCR. J. Gen. Plant Pathol. 78:247-254.

Hamada, M. S., Yin, Y., Chen, H. G., and Ma, Z. 2011. The escalating threat of Rhizoctonia cerealis, the causal agent of sharp eyespot in wheat. Pest Manag. Sci. 67:1411-1419.

Haratian, M., Safaie, N., Sharifnabi, B., Mahmudi, S. B., and Ariana, A. 2013. Genetic structure of populations of Rhizoctonia solani AG-4 from five provinces in Iran. Plant Pathol. 62:649-656.

Hayakawa, T., Toda, T., Ping, Q., Mghalu, J. M., Yaguchi, S., and Hyakumachi, M. 2006. A new subgroup of Rhizoctonia AG-D, AG-D III, obtained from Japanese zoysia grass exhibiting symptoms of a new disease. Plant Dis. 90:1389-1394.

Jakobsson, M., and Rosenberg, N. A. 2007. CLUMPP: A cluster matching and permutation program for dealing with label switching and multimodality in analysis of population structure. Bioinformatics 23:1801-1806.

Li, W., Sun, H., Deng, Y., Zhang, A., and Chen, H. 2014. The heterogeneity of the rDNA-ITS sequence and its phylogeny in Rhizoctonia cerealis, the cause of sharp eyespot in wheat. Curr. Genet. 60:1-9.

Lim, S., Notley-McRobb, L., Lim, M., and Carter, D. A. 2004. A comparison of the nature and abundance of microsatellites in 14 fungal genomes. Fungal Genet. Biol. 41:1025-1036.

Lipps, R. E., and Herr, L. J. 1982. Etiology of Rhizoctonia cerealis in sharp eyespot of wheat. Phytopathology 72:1574-1577.

Manly, B. F. J. 1991. Randomization, Bootstrap and Monte Carlo Methods in Biology, 2nd Ed. Chapman and Hall and CRC, London.

McBeath, J. H., and McBeath, J. 2010. Plant diseases, pests and food security. Page 117 in: Environmental Change and Food Security in China. B. Martin, ed. Springer Technology and Engineering, the Netherlands.
McDonald, B. A., and Linde, C. 2002. Pathogen population genetics, evolutionary potential, and durable resistance. Annu. Rev. Phytopathol. 40: 349-379.

Meirmans, P. G., and Van Tienderen, P. H. 2004. GenoType and GenoDive: Two programs for the analysis of genetic diversity of asexual organisms. Mol. Ecol. Notes 4:792-794.

Milgroom, M. G. 1996. Recombination and the multilocus structure of fungal populations. Annu. Rev. Phytopathol. 34:457-477.

Nei, M. 1978. Estimation of average heterozygosity and genetic distance from a number of individuals. Genetics 89:538-590.

Padasht-Dehkaei, F., Ceresini, P. C., Zala, M., Okhovvat, S. M., Nikkhah, M. J., and McDonald, B. A. 2013. Population genetic evidence that basidiospores play an important role in the disease cycle of rice-infecting populations of Rhizoctonia solani AG-1 IA in Iran. Plant Pathol. 62:49-58.

Pereira, D. A. S., Ceresini, P., Castroagudín, V. L., Ramos Molina, L. M., Chavarro Mesa, E., Negrisoli, M. M., Campos, S. N., Souza Pegolo, M. E., and Takada, H. M. 2016. Population genetic structure of Rhizoctonia oryzae-sativae from rice in Latin America and its adaptive potential to emerge as pathogen on Urochloa pastures. Phytopathology. doi:10.1094/ PHYTO-05-16-0219-R

Pritchard, J. K., Stephens, M., and Donnelly, P. 2000. Inference of population structure using multilocus genotype data. Genetics 155:945-959.

Raymond, M., and Rousset, F. 1995. GENEPOP (version 1.2): Population genetics software for exact tests and ecumenicism. J. Hered. 86:248-249.

Ren, X. R., Li, W., Sun, H. Y., Yu, H. S., and Chen, H. G. 2013. The expression of carbohydrate active enzymes of wheat sharp eyespot pathogen, Rhizoctonia cerealis. Acta Phytopathol. Sin. 43:129.

Smith, J. M., Smith, N. H., O'Rourke, M., and Spratt, B. G. 1993. How clonal are bacteria? Proc. Natl. Acad. Sci. USA 90:4384-4388.

Stoddart, J. A., and Taylor, J. F. 1988. Genotype diversity: Estimation and prediction in samples. Genetics 118:705-711.

Sun, H., Wang, C., Li, W., Zhang, A., Deng, Y., and Chen, H. 2015. Characterization of Rhizoctonia cerealis sensitivity to thifluzamide in China. Crop Prot. 69:65-69.

Toda, T., Hyakumachi, M., Suga, H., Kageyama, K., Tanaka, A., and Tani, T. 1999. Differentiation of Rhizoctonia AG-D isolates from turfgrass into subgroups I and II based on rDNA and RAPD analysis. Eur. J. Plant Pathol. 105:835-846.

Zala, M., McDonald, B. A., Bernardes-de-Assis, J., Ciampi, M. B., Storari, M., Peyer, P., and Ceresini, P. C. 2008. Highly polymorphic microsatellite loci in the maize- and rice-infecting fungal pathogen Rhizoctonia solani anastomosis group 1 IA. Mol. Ecol. Resour. 8:686-689.

Zhan, J., Pettway, R. E., and McDonald, B. A. 2003. The global genetic structure of the wheat pathogen Mycosphaerella graminicola is characterized by high nuclear diversity, low mitochondrial diversity, regular recombination, and gene flow. Fungal Genet. Biol. 38:286-297. 